\title{
Adherence to Rivaroxaban Compared with Other Oral Anticoagulant Agents Among Patients with Nonvalvular Atrial Fibrillation
}

\author{
Colleen A. McHorney, PhD; Veronica Ashton, MPH; François Laliberté, MA; Guillaume Germain, MA; \\ Willy Wynant, PhD; Concetta Crivera, PharmD, MPH; Jeffrey R. Schein, DrPH, MPH; \\ Patrick Lefebvre, MA; and Eric D. Peterson, MD, MPH
}

\begin{abstract}
BACKGROUND: Adherence to oral anticoagulant (OAC) agents is important for patients with nonvalvular atrial fibrillation (NVAF) to prevent potentially severe adverse events.

OBJECTIVE: To compare real-world adherence rates and time to discontinuation for rivaroxaban versus other OACs (apixaban, dabigatran, and warfarin) among patients with NVAF using claims-based data.

METHODS: Health care claims from the IMS Health Real-World Data Adjudicated Claims database (July 2012-June 2015) were analyzed. Adherence rate was defined as the percentage of patients with proportion of days covered $(P D C) \geq 0.80$ and $\geq 0.90$. Discontinuation was defined as a gap of more than 30 days between the end of a dispensing days of supply and the start date of the next fill, if any. Patients were included if they had $\geq 2$ dispensings of rivaroxaban, apixaban, dabigatran, or warfarin at least 180 days apart (the first was considered the index date), had $>60$ days of supply, had $\geq 6$ months of pre-index eligibility, had $\geq 1$ atrial fibrillation (AF) diagnosis pre-index or at index date, and had no valvular involvement. $A$ logistic regression model was used to evaluate adherence to OAC therapy, while a Cox model was used to compare time to discontinuation; both models adjusted for baseline confounders.
\end{abstract}

RESULTS: A total of 13,645 rivaroxaban, 6,304 apixaban, 3,360 dabigatran, and 13,366 warfarin patients were identified. A significantly higher proportion of rivaroxaban users $(80.1 \%)$ was adherent to therapy $(P D C \geq 0.80$ at 6 months) versus apixaban $(75.8 \%)$, dabigatran $(69.2 \%)$, and warfarin users $(64.5 \%)$. After adjustment, the proportion of patients adherent to therapy remained significantly higher for rivaroxaban users versus apixaban (absolute difference $[A D]=5.8 \%)$, dabigatran $(A D=9.5 \%)$, and warfarin users $(\mathrm{AD}=13.6 \%$; all $P<0.001)$. More pronounced differences were found with a $\mathrm{PDC} \geq 0.90$. In addition, rivaroxaban users were significantly less likely to discontinue therapy compared with other OACs after adjustments (all $P<0.05$ ).

CONCLUSIONS: Among NVAF patients, rivaroxaban was associated with significantly higher adherence rates relative to other OACs whether using either a PDC of $>0.80$ or $>0.90$. Such differences in adherence could translate into improved patient outcomes and lower health care costs.

J Manag Care Spec Pharm. 2017;23(9):980-88

Copyright $\odot 2017$, Academy of Managed Care Pharmacy. All rights reserved.

\section{What is already known about this subject}

Poor medication adherence, especially among oral anticoagulants (OACs), has been associated with worse patient outcomes and therapy failure.
Compared with vitamin K anticoagulants, such as warfarin, nonvitamin $\mathrm{K}$ oral anticoagulants (NOACs) do not require recurrent monitoring, have more predictable pharmacokinetics, and have fewer drug interactions.

\section{What this study adds}

This study showed that patients treated with rivaroxaban had a significantly higher adherence to therapy relative to the other NOACs (i.e., apixaban and dabigatran) and warfarin whether using a proportion of days covered threshold of 0.80 or 0.90 to define an adherent patient.

Rivaroxaban users were also less likely to discontinue their therapy compared with other NOAC and warfarin users.

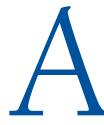
nticoagulant agents are used to prevent blood clots (i.e., stroke prevention) among patients diagnosed with atrial fibrillation (AF) — the most common cardiac arrhythmia in the United States. AF had an estimated U.S. prevalence of 5.2 million in 2010, and total U.S. direct costs attributable to AF have been estimated at $\$ 6.65$ billion annually. ${ }^{1-3} \mathrm{AF}$ is the cause of a substantial public health and economic burden. For example, patients diagnosed with AF have an increased risk of developing adverse events and worse clinical outcomes compared with non-AF patients, such as ischemic stroke, heart failure, chronic fatigue, or additional heart rhythm problems. ${ }^{4-8}$ Furthermore, the risk of AF increases with age-currently more than half of AF patients are aged over 80 years. ${ }^{9,10}$ With the aging of the population, the number of people at risk is expected to rise to 12.1 million by 2030 and lead to a substantial growth of economic burden.,10

Treatment with oral anticoagulant (OAC) agents can substantially reduce the risk of developing adverse events such as strokes and bleedings, reduce the associated risk of morbidity and mortality, and increase or maintain patient quality of life. ${ }^{11}$ Recently, non-vitamin $\mathrm{K}$ oral anticoagulants (NOACs) have been approved by the U.S. Food and Drug Administration for stroke prevention in nonvalvular AF (NVAF) patients. Before the approval of NOAC agents, vitamin $\mathrm{K}$ anticoagulants (VKAs), such as warfarin, were the main treatment options used for stroke prevention among AF patients. ${ }^{12}$ Because VKAs require recurrent monitoring of the anticoagulant effect 
with dose adjustment-and NOACS do not-NOACs offer a less-demanding therapeutic regimen for patients. Moreover, NOACs have more predictable pharmacokinetics and fewer drug interactions. ${ }^{13}$ Currently, licensed NOACs include oncea-day rivaroxaban and edoxaban and twice-a-day dabigatran and apixaban. ${ }^{14-17}$

Safety and effectiveness of therapies depend on patients taking their medications as prescribed, and this is even more important among chronically ill patients. Poor medication adherence, especially with use of OACs, has been associated with worse patient outcomes and therapy failure. ${ }^{18}$ Medication nonadherence is common among NVAF patients, and nonadherence has been estimated to range from $22 \%$ to $58 \%$ among VKA agents. ${ }^{19}$ Because of their recent regulatory approval, there have been limited adherence and persistence data for NOAC agents. ${ }^{20}$

Considering the potential consequences that suboptimal adherence to OACs might have on AF patients, the purpose of this study was to assess real-world medication adherence and persistence to OACs among patients diagnosed with NVAF using U.S. health care claims data.

\section{Methods}

\section{Data Source}

Health insurance claims from the IMS Health Real-World Data (RWD) Adjudicated Claims database between July 1, 2012, and June 30, 2015, were used to conduct the analysis. The IMS RWD Adjudicated Claims database is one of the largest claims databases of U.S. commercial insurers. This de-identified, integrated database includes medical and pharmacy claims for more than 95 million unique U.S. enrollees. Data elements included inpatient and outpatient claims, diagnoses and procedures based on International Classification of Diseases, Ninth Revision, Clinical Modification (ICD-9-CM) and Current Procedural Terminology, 4th Edition (CPT-4) codes, and retail and mail order pharmacy claims. Because of the broad reach of the data, records in the IMS RWD Adjudicated Claims database are representative of the national commercially insured population; however, patients from the western region are underrepresented.

\section{Study Design}

A retrospective cohort design was used to assess adherence to rivaroxaban, apixaban, dabigatran, and warfarin among patients with NVAF. Criteria defined by the Pharmacy Quality Alliance (PQA) were used to identify the study population. ${ }^{21}$ More specifically, patients were included if they had 2 or more dispensings of OACs at least 180 days apart after January 1, 2013 (the date of the first dispensing was considered the index date, and the type of OAC agent was considered the index agent) and had more than 60 days of supply. In addition to the preceding 2 PQA criteria, patients had to be newly initiated on the index anticoagulants agent (i.e., no previous use of the index agent in the 6 months before the index date), had to have a baseline period of at least 6 months of continuous health plan enrollment before the index date, and had to have at least 1 primary or secondary AF diagnosis (ICD-9-CM code 427.31) during the baseline period or at the index date. Patients were excluded if they were aged under 18 years at the index date or if they were diagnosed at baseline with valvular involvement (including ICD-9-CM codes 394.0x, 394.2x, 396.0x, 396.1x, 746.5x, 996.02, 996.71 for mitral stenosis diagnosis; ICD-9Procedure codes 35.20, 35.22, 35.23, 35.24, 35.97 and CPT-4 codes 33405, 33420, 33422, 33425-33427, 33430, 92987 for mechanical heart valves).

The observation period spanned from the index date to the end of insurance coverage, end of data availability (i.e., June 30,2015), or a potentially curative procedure (i.e., catheter ablation and surgical maze; ICD-9-Procedure code 37.34 or CPT-4 codes 93650-93652, 93799, 33250-33251, 3325433259, 33261, 33265-33266), whichever occurred first.

\section{Study Endpoints}

Adherence to OACs was evaluated using proportion of days covered (PDC) at 6 months and medication possession ratio (MPR). PDC was calculated as the number of days of supply over a fixed period of time (i.e., 6 months). MPR was calculated as the number of days of supply over treatment duration (i.e., the period between the first and the last dispensing of the index medication). Adherence to therapy was defined using PDC or MPR thresholds of $\geq 0.80$ and $\geq 0.90$.

Discontinuation was evaluated at 3, 6, 9, and 12 months and was defined as a gap of more than 30 days between the end of a dispensing days of supply and the start date of the next fill, if any. Sensitivity analyses were conducted using gaps of more than 15 and 45 days to define discontinuation.

\section{Statistical Analysis}

Descriptive statistics of cohorts were generated to summarize patient baseline characteristics and risk factors evaluated during the 6 months before the index date. Means, medians, and standard deviations (SDs) were used to describe continuous variables, while frequencies and percentages were used to describe categorical variables. Student's t-tests and chi-squared tests were performed to compare adherence between cohorts. In addition, treatment patterns (i.e., the number of dispensings and days of supply of the index drug) were reported over the observation period using means and SDs.

Adherence to the index therapy was reported using PDC and MPR at 6 months. The proportion of patients with PDCs (and MPR) $\geq 0.80$ and $\geq 0.90$ were reported. Comparisons between cohorts were assessed using unadjusted and adjusted logistic regression models. Adjustments included the following sociodemographic and clinical characteristics: age; gender; region; insurance type; index month and year; previous $\mathrm{OAC}$ use; Quan-Charlson Comorbidity Index score; $\mathrm{CHA}_{2} \mathrm{DS}_{2}$-VASc 


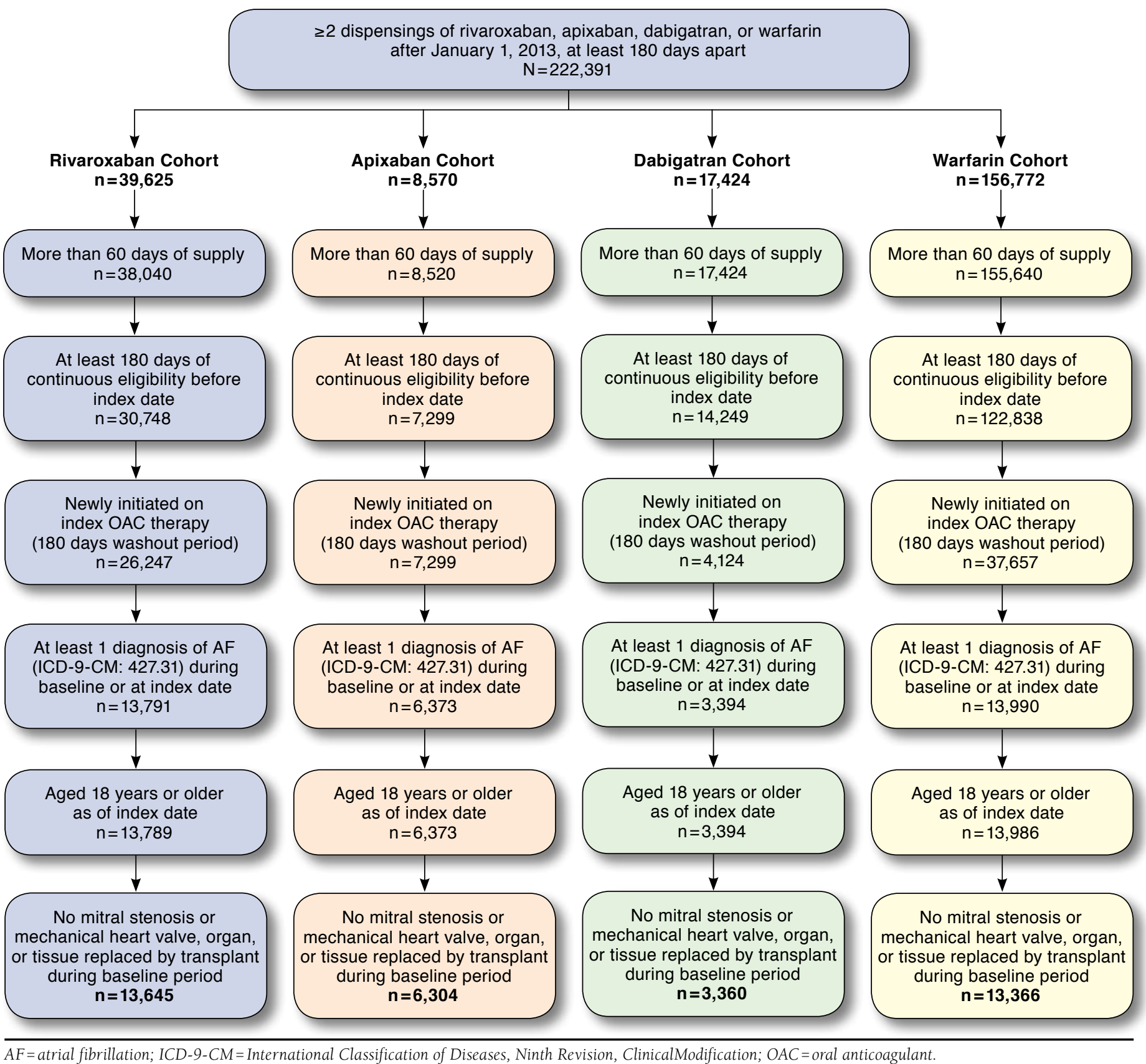

(i.e., a clinical prediction rule estimating the risk of stroke in patients diagnosed with $\mathrm{AF}$ ) and HAS-BLED (i.e., a scoring system assessing the 1-year risk of major bleeding in patients diagnosed with AF) scores; previous diagnosis of venous thromboembolism, total hip arthroplasty, or total knee arthroplasty; and the number of different drug classes used at baseline.
Furthermore, adjustments were made for stroke and bleeding risk factors, as well as the following risk factors for nonadherence (defined as mental disorders [depressive disorders, schizophrenia spectrum and other psychotic disorders, bipolar and related disorders, anxiety disorders, obsessive-compulsive and related disorders, dissociative disorders, somatic 
TABLE 1 Demographics and Clinical Characteristics

\begin{tabular}{|c|c|c|c|c|}
\hline & $\begin{array}{l}\text { Rivaroxaban Cohort } \\
\quad(n=13,645)\end{array}$ & $\begin{array}{l}\text { Apixaban Cohort } \\
\quad(n=6,304)\end{array}$ & $\begin{array}{l}\text { Dabigatran Cohort } \\
\quad(n=3,360)\end{array}$ & $\begin{array}{l}\text { Warfarin Cohort } \\
\quad(n=13,366)\end{array}$ \\
\hline Observation period, ${ }^{\text {a }}$ days, mean \pm SD [median] & $493 \pm 190[467]$ & $423 \pm 161[391]$ & $548 \pm 202[547]$ & $544 \pm 201[536]$ \\
\hline \multicolumn{5}{|l|}{ Demographics ${ }^{b}$} \\
\hline Age, years, mean \pm SD [median] & $63.1 \pm 10.0 \quad[62]$ & $64.2 \pm 9.9 \quad[63]$ & $62.9 \pm 9.6 \quad[62]$ & $66.1 \pm 11.0 \quad[64]$ \\
\hline Gender, female, n (\%) & $4,264(31.2)$ & $2,049(32.5)$ & $1,000(29.8)$ & $4,615(34.5)$ \\
\hline \multicolumn{5}{|l|}{ Region, ${ }^{b}$ n (\%) } \\
\hline South & $5,149(37.7)$ & $2,882(45.7)$ & $1,237(36.8)$ & $3,454(25.8)$ \\
\hline West & $863 \quad(6.3)$ & $381 \quad(6.0)$ & $243(7.2)$ & $1,391(10.4)$ \\
\hline Northeast & $3,837(28.1)$ & $1,303(20.7)$ & $980(29.2)$ & $3,976(29.7)$ \\
\hline Midwest & $3,796(27.8)$ & $1,738(27.6)$ & $900(26.8)$ & $4,545(34.0)$ \\
\hline \multicolumn{5}{|l|}{ Insurance type, ${ }^{b}$ n (\%) } \\
\hline $\mathrm{PPO}$ & $10,645(78.0)$ & $5,115(81.1)$ & $2,569(76.5)$ & $9,219(69.0)$ \\
\hline $\mathrm{HMO}$ & $1,551(11.4)$ & $568 \quad(9.0)$ & $402(12.0)$ & $2,421(18.1)$ \\
\hline Indemnity/traditional & $660 \quad(4.8)$ & $295 \quad(4.7)$ & $159 \quad(4.7)$ & $899 \quad(6.7)$ \\
\hline POS & $499 \quad(3.7)$ & $184 \quad(2.9)$ & $161 \quad(4.8)$ & $536 \quad(4.0)$ \\
\hline $\mathrm{CDHP}$ & $90 \quad(0.7)$ & $36 \quad(0.6)$ & $17 \quad(0.5)$ & $68 \quad(0.5)$ \\
\hline HSA & $2(0.0)$ & $0 \quad(0.0)$ & $1 \quad(0.0)$ & $1 \quad(0.0)$ \\
\hline Unknown & $198 \quad(1.5)$ & $106 \quad(1.7)$ & $51 \quad(1.5)$ & $222(1.7)$ \\
\hline \multicolumn{5}{|l|}{ Comorbidity index scores, ${ }^{\mathrm{c}}$ mean \pm SD [median] } \\
\hline Quan-Charlson Comorbidity Index & $1.48 \pm 1.68[1]$ & $1.58 \pm 1.72[1]$ & $1.40 \pm 1.56[1]$ & $2.04 \pm 2.08[1]$ \\
\hline $\mathrm{CHA}_{2} \mathrm{DS}_{2}$-VASc score & $2.35 \pm 1.40[2]$ & $2.48 \pm 1.39[2]$ & $2.29 \pm 1.38[2]$ & $2.77 \pm 1.51[3]$ \\
\hline HAS-BLED score & $1.53 \pm 1.05[1]$ & $1.62 \pm 1.03[2]$ & $1.50 \pm 1.00[1]$ & $1.79 \pm 1.15[2]$ \\
\hline \multicolumn{5}{|l|}{ Comorbidity ${ }^{\mathrm{c}}$} \\
\hline VTE & $976 \quad(7.2)$ & $180 \quad(2.9)$ & $66 \quad(2.0)$ & $1,434(10.7)$ \\
\hline THA/TKA & $305 \quad(2.2)$ & $96 \quad(1.5)$ & $54 \quad(1.6)$ & $288(2.2)$ \\
\hline \multicolumn{5}{|l|}{ Baseline medicationc } \\
\hline Number of different drug classes & $6.81 \pm 4.06[6]$ & $7.39 \pm 4.10[7]$ & $6.59 \pm 4.05[6]$ & $6.59 \pm 4.20[6]$ \\
\hline \multicolumn{5}{|l|}{ Baseline use of oral anticoagulant } \\
\hline Any oral anticoagulant & $3,950(28.9)$ & $2,083(33.0)$ & $644(19.2)$ & $1,004 \quad(7.5)$ \\
\hline Warfarin & $2,877(21.1)$ & $1,230(19.5)$ & $539(16.0)$ & $0 \quad(0.0)$ \\
\hline Rivaroxaban & $0 \quad(0.0)$ & $446 \quad(7.1)$ & $103(3.1)$ & $515 \quad(3.9)$ \\
\hline Dabigatran & $1,063 \quad(7.8)$ & $481 \quad(7.6)$ & $0 \quad(0.0)$ & $439 \quad(3.3)$ \\
\hline Apixaban & $86 \quad(0.6)$ & $0 \quad(0.0)$ & $14 \quad(0.4)$ & $95 \quad(0.7)$ \\
\hline Edoxaban & $0 \quad(0.0)$ & $0 \quad(0.0)$ & $0 \quad(0.0)$ & $0 \quad(0.0)$ \\
\hline \multicolumn{5}{|l|}{ Baseline resource utilization, ${ }^{\mathrm{c}}$ mean \pm SD [median] } \\
\hline Hospitalizations & $0.45 \pm 0.66[0]$ & $0.42 \pm 0.65[0]$ & $0.39 \pm 0.61[0]$ & $0.55 \pm 0.75[0]$ \\
\hline Hospital days & $2.96 \pm 9.58[0]$ & $2.52 \pm 8.19[0]$ & $2.22 \pm 7.86[0]$ & $6.20 \pm 16.56[0]$ \\
\hline ER visits & $0.42 \pm 0.92[0]$ & $0.46 \pm 1.14[0]$ & $0.38 \pm 0.96[0]$ & $0.46 \pm 1.11[0]$ \\
\hline Outpatient visits & $11.31 \pm 10.59[9]$ & $12.16 \pm 11.00[9]$ & $10.37 \pm 9.58[8]$ & $13.24 \pm 13.32[10]$ \\
\hline \multicolumn{5}{|l|}{ Baseline health care cost, ${ }^{c}$ \$US 2015 , mean \pm SD } \\
\hline Total health care cost & $15,587 \pm 42,340$ & $15,623 \pm 33,304$ & $12,661 \pm 28,618$ & $32,451 \pm 179,458$ \\
\hline Hospitalizations & $8,871 \pm 39,020$ & $7,623 \pm 26,864$ & $6,893 \pm 25,459$ & $24,073 \pm 151,960$ \\
\hline ER visits & $387 \pm 2,906$ & $392 \pm 1,769$ & $328 \pm 2,177$ & $1,034 \pm 72,948$ \\
\hline Outpatient visits & $4,856 \pm 12,109$ & $6,022 \pm 17,082$ & $4,116 \pm 10,351$ & $5,942 \pm 27,004$ \\
\hline Pharmacy & $1,472 \pm 3,373$ & $1,585 \pm 2,868$ & $1,321 \pm 3,067$ & $1,400 \pm 4,823$ \\
\hline $\begin{array}{l}\text { Index medication copayment, }{ }^{b} \$ \text { US } 2015, \\
\text { mean } \pm \text { SD [median] }\end{array}$ & $40.32 \pm 52.76[32]$ & $46.06 \pm 52.95[41]$ & $42.89 \pm 51.64[36]$ & $6.10 \pm 10.31[4]$ \\
\hline \multicolumn{5}{|c|}{ Comorbidities, substance abuse, stress and social risk factors associated with nonadherence, $\mathrm{n}(\%) \mathrm{c}, \mathrm{d}$} \\
\hline 0 & $8,195(60.1)$ & $3,774(59.9)$ & $2,040(60.7)$ & $8,188(61.3)$ \\
\hline 1 & $3,956(29.0)$ & $1,926(30.6)$ & $960(28.6)$ & $3,896(29.1)$ \\
\hline$\geq 2$ & $1,494(10.9)$ & $604 \quad(9.6)$ & $360(10.7)$ & $1,282 \quad(9.6)$ \\
\hline \multicolumn{5}{|l|}{ Frequent risk factors, ${ }^{\mathrm{c}, \mathrm{e}} \mathbf{n}(\%)$} \\
\hline Hypertension & $9,694(71.0)$ & $4,736(75.1)$ & $2,423(72.1)$ & $9,453(70.7)$ \\
\hline Hyperlipidemia & $7,671(56.2)$ & $3,781(60.0)$ & $1,889(56.2)$ & $7,134(53.4)$ \\
\hline
\end{tabular}


TABLE 1 Demographics and Clinical Characteristics (continued)

\begin{tabular}{|c|c|c|c|c|}
\hline & $\begin{array}{c}\text { Rivaroxaban Cohort } \\
\quad(n=13,645)\end{array}$ & $\begin{array}{l}\text { Apixaban Cohort } \\
(\mathrm{n}=6,304)\end{array}$ & $\begin{array}{l}\text { Dabigatran Cohort } \\
\quad(\mathbf{n}=3,360)\end{array}$ & $\begin{array}{l}\text { Warfarin Cohort } \\
\quad(n=13,366)\end{array}$ \\
\hline \multicolumn{5}{|c|}{ Frequent risk factors, ${ }^{\mathrm{c}, \mathrm{e}} \mathbf{n}(\%)$} \\
\hline Coronary heart disease & $4,420(32.4)$ & $2,328(36.9)$ & $1,048(31.2)$ & $5,124(38.3)$ \\
\hline Diabetes & $3,743(27.4)$ & $1,795(28.5)$ & $987(29.4)$ & $4,130(30.9)$ \\
\hline Heart failure & $2,725(20.0)$ & $1,320(20.9)$ & $639(19.0)$ & $3,727(27.9)$ \\
\hline \multicolumn{5}{|c|}{ 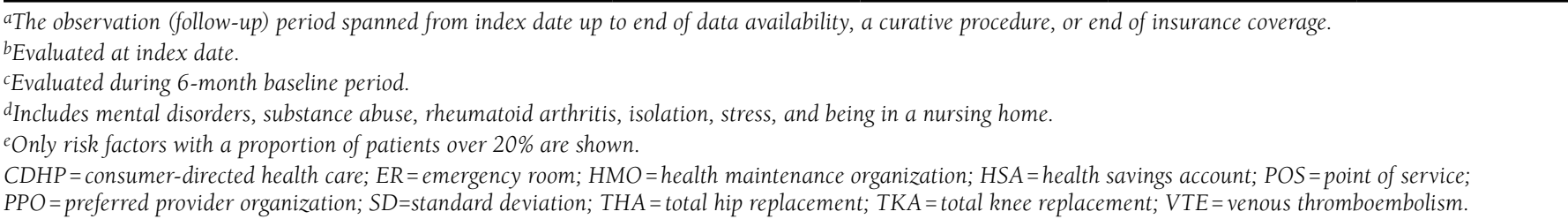 } \\
\hline
\end{tabular}

TABLE 2 Adherence Measures of OAC Cohorts

\begin{tabular}{|c|c|c|c|c|c|c|c|}
\hline \multirow{2}{*}{$\begin{array}{l}\text { Treatment Patterns and } \\
\text { Adherence }\end{array}$} & \multirow{2}{*}{$\begin{array}{c}\text { Rivaroxaban Cohort } \\
{[\mathrm{A}]} \\
(\mathrm{n}=13,645)\end{array}$} & \multirow{2}{*}{$\begin{array}{c}\text { Apixaban Cohort } \\
{[\mathrm{B}]} \\
(\mathrm{n}=6,304)\end{array}$} & \multirow{2}{*}{$\begin{array}{c}\text { Dabigatran Cohort } \\
{[C]} \\
(\mathrm{n}=3,360)\end{array}$} & \multirow{2}{*}{$\begin{array}{c}\text { Warfarin Cohort } \\
{[\mathrm{D}]} \\
(\mathrm{n}=13,366)\end{array}$} & \multicolumn{3}{|c|}{$P$ Value ${ }^{a}$} \\
\hline & & & & & {$[\mathrm{A}]$ vs. $[\mathrm{B}]$} & {$[\mathrm{A}]$ vs. $[\mathrm{C}]$} & [A] vs. [D] \\
\hline $\begin{array}{l}\text { Treatment duration, days, } \\
\text { mean (SD) }\end{array}$ & $452(183)$ & $402(156)$ & $485(196)$ & $496(196)$ & - & - & - \\
\hline $\begin{array}{l}\text { Number of dispensing per } \\
\text { patient, mean (SD) }\end{array}$ & $11.41(6.0)$ & $10.50(5.2)$ & $11.05(6.2)$ & $11.31(8.8)$ & - & - & - \\
\hline $\begin{array}{l}\text { Days supply per } \\
\text { dispensing, mean (SD) }\end{array}$ & $44.44(24.1)$ & $41.68(21.9)$ & $45.35(24.4)$ & $59.87(41.1)$ & - & - & - \\
\hline \multicolumn{8}{|l|}{$\mathrm{MPR}^{\mathrm{b}}$} \\
\hline MPR, mean (SD) & $0.86(0.2)$ & $0.85(0.2)$ & $0.81(0.2)$ & $0.80(0.2)$ & 0.001 & $<0.001$ & $<0.001$ \\
\hline $\begin{array}{l}\text { Adherent patients, \% } \\
(\mathrm{MPR} \geq 0.80)\end{array}$ & 78.1 & 75.7 & 66.4 & 59.0 & $<0.001$ & $<0.001$ & $<0.001$ \\
\hline $\begin{array}{l}\text { Adherent patients, \% } \\
(\mathrm{MPR} \geq 0.90)\end{array}$ & 57.6 & 53.0 & 45.5 & 37.2 & $<0.001$ & $<0.001$ & $<0.001$ \\
\hline \multicolumn{8}{|c|}{ Unadjusted adherence at 6 months ${ }^{c}$} \\
\hline$\%$ of patients with $\mathrm{PDC} \geq 0.80$ & 80.1 & 75.8 & 69.2 & 64.5 & $<0.001$ & $<0.001$ & $<0.001$ \\
\hline$\%$ of patients with $P D C \geq 0.90$ & 63.5 & 56.9 & 51.3 & 47.1 & $<0.001$ & $<0.001$ & $<0.001$ \\
\hline \multicolumn{8}{|c|}{$\begin{array}{l}\text { aP values were estimated using Student's t-tests for continuous variables and chi-squared tests for categorical variables. } \\
\text { bDefined as the number of days supply between the first and the last dispensing over treatment duration. Treatment duration spanned from index date until earliest date } \\
\text { between end of data availability, a curative procedure, and end of insurance coverage. } \\
\text { cUnadusted adherence was calculated as the proportion of patients with } P D C \geq 0.80 \text { or } P D C \geq 0.90 \text {. } \\
M P R=\text { medication possession ratio; } P D C=\text { proportion of days covered; } S D=\text { standard deviation. }\end{array}$} \\
\hline
\end{tabular}

symptoms and related disorders, feeding and eating disorders, neurodevelopmental disorders, sleep-wake disorders, neurocognitive disorders, medication-induced movement disorders, and other adverse effect medication and personality disorders]; substance abuse; diagnosis of rheumatoid arthritis; isolation; stress; total copayment at index; and total health care costs). Statistical significance was assessed at an a-level of 0.05 or less.

Kaplan-Meier rates of persistence were evaluated for each cohort, and multivariable Cox proportional hazards regression models were used to compare the time to discontinuation between different OAC users, adjusting for baseline confounders (i.e., the same covariates as for adherence analyses were used). Hazard ratios (HRs) and corresponding 95\% confidence intervals (CIs) from the Cox models were used to compare cohorts.
All statistical analyses were conducted using SAS version 9.3 (SAS Institute, Cary, NC).

\section{Results}

\section{Patient Characteristics}

Of all NVAF patients ( $\mathrm{N}=222,453), 13,645$ rivaroxaban, 6,304 apixaban, 3,360 dabigatran, and 13,366 warfarin users met the inclusion and exclusion criteria to form the final study cohorts (Figure 1). Table 1 summarizes baseline characteristics of each study cohort. The mean (SD) observation period was 493 (190), 423 (161), 548 (202), and 544 (201) days for the rivaroxaban, apixaban, dabigatran, and warfarin cohorts, respectively. The mean age was similar between the 3 NOAC cohorts at 63.1, 


\section{A. Percentage of Subjects with $P D C \geq 0.80$ at 6 Months}

Rivaroxaban versus:

Apixaban: absolute difference $=5.8 \% ; P<0.001$

Dabigratran: absolute difference $=9.5 \% ; P<0.001$

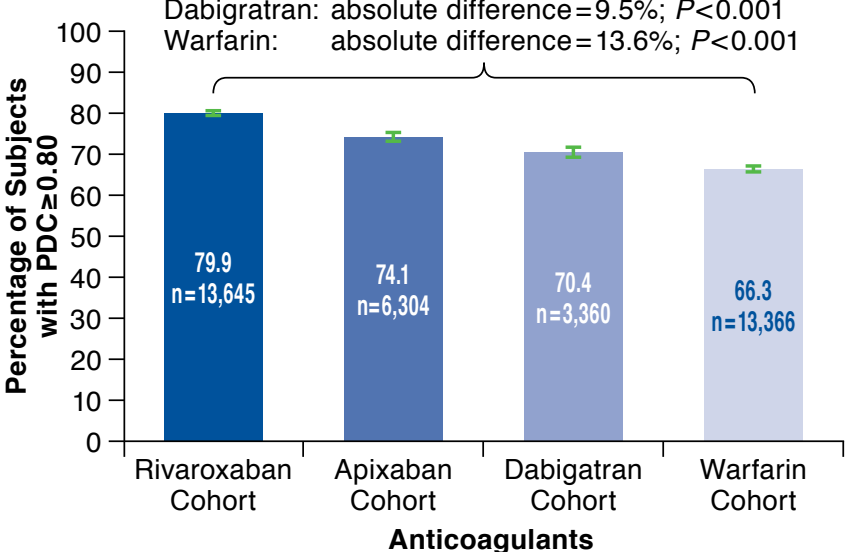

\section{B. Percentage of Subjects with PDC $\geq 0.90$ at 6 Months}

Rivaroxaban versus:

Apixaban: absolute difference $=8.0 \% ; P<0.001$

Dabigratran: absolute difference $=11.2 \% ; P<0.001$

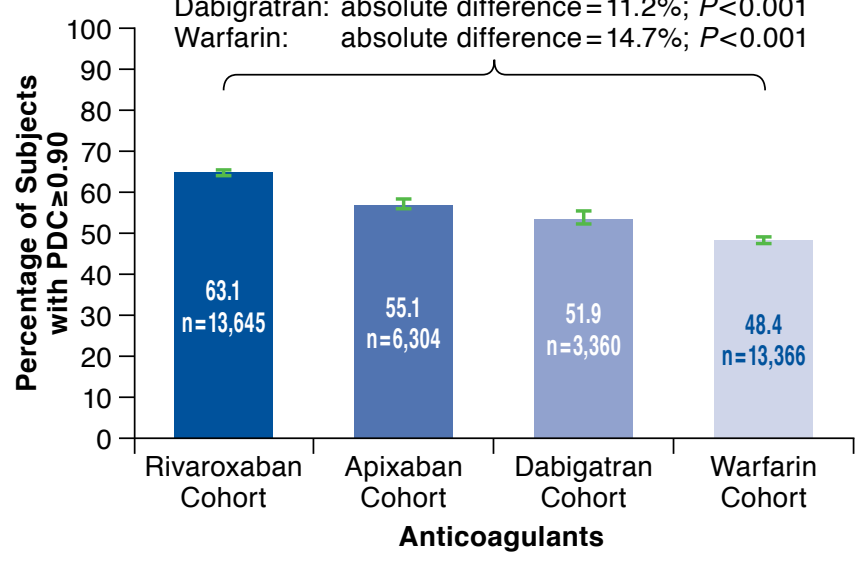

aAdjusted adherence was calculated as the predicted probability of $P D C \geq 0.80$ or $P D C \geq 0.90$ based on multivariable logistic regression. $O A C=$ oral anticoagulant $; P C=$ proportion of days covered.

64.2 , and 62.9 years for the rivaroxaban, apixaban, and dabigatran cohorts, respectively, while warfarin patients were slightly older (mean age of 66.1 years). The most prevalent risk factors were hypertension (71\%-75\%), hyperlipidemia (53\%-60\%), coronary heart disease (31\%-38\%), and diabetes (27\%-31\%).

On average, the NOAC cohorts were similar; however, notable differences existed at baseline between warfarin users and NOAC users. The warfarin cohort had higher Quan-Charlson Comorbidity Index, $\mathrm{CHA}_{2} \mathrm{DS}_{2}$-VASc, and HAS-BLED scores than the rivaroxaban, apixaban, and dabigatran cohorts (all $P$ values $<0.001$, data not shown). The warfarin cohort also had more hospitalizations and higher total hospitalization costs relative to each NOAC cohort during the 6-month baseline period. Of note, NOAC users were more likely to have previously used anticoagulant agents than warfarin users (all $P$ values $<0.001$, data not shown).

\section{Treatment Patterns and Medication Adherence}

Table 2 presents the treatment patterns and the unadjusted adherence results of rivaroxaban, apixaban, dabigatran, and warfarin users. The proportion of patients with $P D C \geq 0.80$ at 6 months was significantly higher in the rivaroxaban cohort (80.1\%) compared with the apixaban $(75.8 \%)$, dabigatran (69.2\%), and warfarin (64.5\%) cohorts (all $P<0.001$ ). The proportion of patients with $\mathrm{PDC} \geq 0.90$ was $63.5 \%, 56.9 \%$, $51.3 \%$, and $47.1 \%$ for the rivaroxaban, apixaban, dabigatran, and warfarin cohorts, respectively. The mean MPR was significantly higher in the rivaroxaban cohort (0.86) compared with the apixaban $(0.85, P=0.001)$, dabigatran $(0.81, P<0.001)$, and warfarin $(0.80, P<0.001)$ cohorts.

After adjusting for baseline demographics and clinical characteristics, the proportion of patients with PDC $\geq 0.80$ was $79.9 \%$, $74.1 \%, 70.4 \%$, and $66.3 \%$ for rivaroxaban, apixaban, dabigatran, and warfarin users, respectively (Figure 2A). The associated absolute differences (ADs) in proportion of patients adherent to therapy remained significantly higher for rivaroxaban users versus apixaban $(\mathrm{AD}=5.8 \%)$, dabigatran $(\mathrm{AD}=9.5 \%)$, and warfarin users $(\mathrm{AD}=13.6 \%$, all $P<0.001$; Figure $2 \mathrm{~A}$ and Appendix, available in online article). Similarly, the adjusted proportion of patients with $\mathrm{PDC} \geq 0.90$ was $63.1 \%, 55.1 \%, 51.9 \%$, and $48.4 \%$ for the rivaroxaban, apixaban, dabigatran, and warfarin cohorts, respectively, which represented even more pronounced ADs between rivaroxaban users and apixaban $(\mathrm{AD}=8.0 \%)$, dabiga$\operatorname{tran}(\mathrm{AD}=11.2 \%)$, and warfarin users $(\mathrm{AD}=14.7 \%$, all $P<0.001$; Figure 2B).

\section{Treatment Persistence and Time to Discontinuation}

Table 3 presents Kaplan-Meier rates of persistence to therapy for rivaroxaban, apixaban, dabigatran, and warfarin users evaluated at 3, 6, 9, and 12 months; these rates showed a higher persistence for rivaroxaban users compared with other OAC users at all time points evaluated. Patients treated with rivaroxaban were also significantly less likely to discontinue therapy at 3, 6, 9, and 12 months compared with those treated 
TABLE 3 Kaplan-Meier Rates of Persistence and Time to Discontinuation Between Rivaroxaban and Other OAC Agents

\begin{tabular}{|c|c|c|c|c|c|c|c|c|}
\hline & & & Surviva & Function & & Ad & Hazard Ratios (9 & \\
\hline Adhe & rence & $\begin{array}{c}\text { Rivaroxaban } \\
\text { Cohort } \\
{[\mathrm{A}]} \\
(\mathrm{n}=13,645) \\
(\%)\end{array}$ & $\begin{array}{c}\text { Apixaban } \\
\text { Cohort } \\
{[\mathrm{B}]} \\
(\mathrm{n}=6,304) \\
(\%)\end{array}$ & $\begin{array}{c}\text { Dabigatran } \\
\text { Cohort } \\
{[\mathrm{C}]} \\
(\mathbf{n}=3,360) \\
(\%)\end{array}$ & $\begin{array}{c}\text { Warfarin } \\
\text { Cohort } \\
{[\mathrm{D}]} \\
(\mathrm{n}=13,366) \\
(\%)\end{array}$ & {$[\mathrm{A}]$ vs. $[\mathrm{B}]$} & {$[\mathrm{A}]$ vs. $[\mathrm{C}]$} & {$[\mathrm{A}]$ vs. [D] } \\
\hline Time & period after & ndex date & & & & & & \\
\hline$n$ & 3 months & 86.0 & 83.4 & 77.3 & 75.3 & $0.78(0.72-0.84)^{b}$ & $0.64(0.59-0.70)^{b}$ & $0.61(0.57-0.65)^{b}$ \\
\hline 践 & 6 months & 78.2 & 75.8 & 67.2 & 62.6 & $0.83(0.78-0.89)^{b}$ & $0.67(0.62-0.71)^{\mathrm{b}}$ & $0.59(0.56-0.63)^{\mathrm{b}}$ \\
\hline$\circ 0$ & 9 months & 69.3 & 68.1 & 57.8 & 51.5 & $0.88(0.83-0.93)^{b}$ & $0.70(0.66-0.74)^{b}$ & $0.61(0.58-0.64)^{b}$ \\
\hline$\wedge$ & 12 months & 59.7 & 59.0 & 47.8 & 41.2 & $0.91(0.86-0.95)^{b}$ & $0.71(0.67-0.75)^{b}$ & $0.63(0.60-0.65)^{b}$ \\
\hline Time & period after & ndex date & & & & & & \\
\hline$n$ & 3 months & 76.1 & 70.7 & 65.6 & 61.5 & $0.77(0.72-0.82)^{\mathrm{b}}$ & $0.69(0.65-0.74)^{b}$ & $0.64(0.61-0.68)^{b}$ \\
\hline สี స్ర & 6 months & 63.4 & 58.4 & 51.1 & 43.7 & $0.82(0.78-0.86)^{b}$ & $0.71(0.67-0.75)^{b}$ & $0.61(0.59-0.64)^{\mathrm{b}}$ \\
\hline$\gamma^{\circ}$ in & 9 months & 51.4 & 48.3 & 39.7 & 31.9 & $0.86(0.82-0.90)^{b}$ & $0.73(0.70-0.77)^{b}$ & $0.63(0.61-0.65)^{b}$ \\
\hline & 12 months & 40.2 & 38.6 & 29.8 & 22.6 & $0.89(0.86-0.93)^{b}$ & $0.75(0.71-0.78)^{\mathrm{b}}$ & $0.64(0.62-0.67)^{\mathrm{b}}$ \\
\hline Time & period after & ndex date & & & & & & \\
\hline & 3 months & 90.4 & 89.9 & 84.5 & 82.3 & $0.86(0.78-0.95)^{b}$ & $0.68(0.61-0.75)^{b}$ & $0.60(0.56-0.65)^{\mathrm{b}}$ \\
\hline 践 & 6 months & 85.1 & 85.1 & 76.7 & 73.1 & $0.89(0.82-0.97)^{b}$ & $0.68(0.62-0.74)^{b}$ & $0.60(0.56-0.64)^{b}$ \\
\hline ำ & 9 months & 78.0 & 79.1 & 68.6 & 63.9 & $0.95(0.89-1.02)$ & $0.71(0.66-0.76)^{b}$ & $0.63(0.59-0.66)^{\mathrm{b}}$ \\
\hline & 12 months & 69.9 & 71.9 & 59.0 & 54.2 & $0.98(0.92-1.04)$ & $0.72(0.68-0.77)^{b}$ & $0.64(0.61-0.68)^{b}$ \\
\hline
\end{tabular}

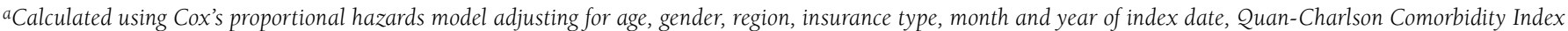

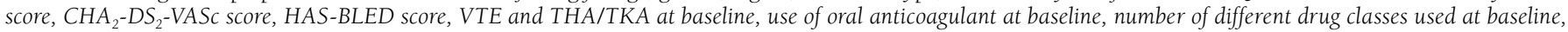
baseline total health care cost, index medication copayment, number of factors associated with nonadherence, and risk factors for bleeding and strokes.

${ }^{b} P \leq 0.05$.

$C I=$ confidence interval; $O A C=$ oral anticoagulant; $T H A=$ total hip replacement; TKA= total knee replacement; VTE=venous thromboembolism .

with other OAC agents (Table 3). Similar discontinuation patterns were observed in sensitivity analyses using gaps of more than 15 and 45 days to define discontinuation, since rivaroxaban users were significantly less likely to discontinue therapy compared with other OAC users (all $P$ values $<0.05$; except rivaroxaban vs. apixaban at 9 and 12 months in the gaps of more than 45 days sensitivity analysis).

\section{Discussion}

This study assessed real-world medication adherence to OAC agents (rivaroxaban, apixaban, dabigatran, and warfarin) among patients with NVAF using U.S. health care claims data from July 2012 to June 2015. Rivaroxaban was associated with significantly higher adherence rates relative to other OACs whether using an unadjusted or adjusted PDC of $\geq 0.80$. For adverse events with potentially serious consequences, such as a stroke, a stricter threshold may be considered to define adherence to therapy. More pronounced differences in adherence were found with an adherence threshold of 0.90. Rivaroxaban users were also found to be less likely to discontinue their therapy compared with other OAC users.
Recent studies have evaluated medication adherence of chronic NOAC users as defined by PQA criteria, and a 3-5 percentage point difference was found in the proportion of patients adherent to therapy in favor of rivaroxaban users compared with apixaban; a corresponding 5-8 percentage point difference was found in favor of rivaroxaban compared with dabigatran users. ${ }^{22}$ Some studies have shown a similar or higher adherence to apixaban than rivaroxaban; however, there were key differences in study designs that may explain this. ${ }^{23,24}$ Studies with single-center, trial-like designs may not represent real-world practice, since they have direct patient support throughout the study follow-up. Consequently, they have significantly higher adherence rates compared with multicenter observational studies. ${ }^{23}$ Also, a study where apixaban compared favorably with the other NOAC agents did not balance the observed time period between cohorts, which increased the risk of bias when comparing adherence. ${ }^{24}$ Comparison of adherence between drugs approved at different points in time may lead to biased results in favor of the newest agent, which was the case with apixaban. Moreover, requiring patients to have at least 2 dispensings, as was the case in our study, would increase the likelihood of accurately measuring adherence in chronic anticoagulant users. 
Dosing frequency and dosing complexity have been identified as factors affecting medication adherence, and reducing dosage frequency from twice to once daily has been associated with improved adherence to therapy. ${ }^{25-27}$ Among medications evaluated in this study, rivaroxaban has a lower pill burdenonce daily-than dabigatran and apixaban, which have twice daily dosing regimens. This difference in dosing frequency may partially explain the higher adherence to therapy of rivaroxaban users compared with other NOAC users. VKA agents also have disadvantages: therapeutic doses between patients can vary greatly, and there is a need for routine monitoring of anticoagulant activity. The burden of monitoring VKA agents could explain the higher adherence rates observed in the NOAC cohorts relative to warfarin users.

\section{Limitations}

This retrospective analysis is subject to limitations. First, adherence measures depend on the service date and days of supply from prescription claim records. In the absence of additional clinical information, it cannot be confirmed if all tablets supplied were actually taken as prescribed. Second, claims data depend on reimbursement coding of health care providers, and billing inaccuracies or omissions in coded procedures, diagnoses, or pharmacy claims are possible. Third, unobservable confounders may have existed that could have resulted in biased estimates. For example, the effect of the use of coupons, adherence intervention programs, or the days of supply of the formulary coverage (e.g., 30 days or 90 days) could not be accounted for in this study. In addition, our adjustment for the copayment at the index date might not represent the overall patient cost share, which has been documented to affect adherence to medication. ${ }^{28}$ Fourth, the observational design of the study is susceptible to additional potential biases such as information or classification bias (e.g., identification of falsepositive or false-negative AF events). Finally, generalization of the study findings could be limited, since the database was predominantly sourced from commercially insured plan members.

\section{Conclusions}

Based on insurance claims data, this large retrospective cohort study showed that patients treated with rivaroxaban had significantly higher adherence to therapy relative to other NOACs (i.e., apixaban and dabigatran) and warfarin whether using a PDC threshold of 0.80 or 0.90 to define an adherent patient. Rivaroxaban users were also less likely to discontinue their therapy compared with other NOAC and warfarin users. Further research is warranted to evaluate the potential effect that these differences may have on patient outcomes and health care resources.

\section{Authors}

COLLEEN A. MCHORNEY, PhD, Evidera, Bethesda, Maryland. VERONICA ASHTON, MPH; CONCETTA CRIVERA, PharmD, MPH; and JEFFREY R. SCHEIN, DrPh, MPH, Janssen Scientific Affairs, Titusville, New Jersey. FRANÇOIS LALIBERTÉ, MA; GUILLAUME GERMAIN, MA; WILLY WYNANT, PhD; and PATRICK LEFEBVRE, MA, Groupe d'analyse, Ltée, Montréal, Quebec, Canada. ERIC D. PETERSON, MD, MPH, Duke Clinical Research Institute, Durham, North Carolina.

AUTHOR CORRESPONDENCE: Guillaume Germain, MA, Economist, Groupe d'analyse, Ltée, 1000 rue de la Gauchetière Ouest, Bureau 1200, Montréal, Québec H3B 4W5 Canada. Tel.: 514.394.4446; E-mail: guillaume.germain@analysisgroup.com.

\section{DISCLOSURES}

This research was funded by Janssen Scientific Affairs. Ashton, Crivera, and Schein are employees and stockholders of Janssen Scientific Affairs. Laliberté, Germain, Wynant, and Lefebvre are employees of Analysis Group, a consulting company that received research grants from Janssen Scientific Affairs in connection with this study. McHorney is an employee of Evidera, a consulting company that received research grants from Janssen Scientific Affairs in connection with this study. Peterson received research grants from Janssen Scientific Affairs in connection with this study.

All authors contributed to concept and design. The data were collected by Germain, Wynant, Laliberté, and Lefebvre and interpreted primarily by McHorney and Peterson, with the assistance of Lefebvre, Laliberté, Ashton, Crivera, and Schein. The manuscript was written primarily by Laliberté, Germain, and Lefebvre, with the assistance of Wynant. Revisions were made primarily by Ashton, Crivera, McHorney, Schein, and Peterson.

\section{REFERENCES}

1. Colilla S, Crow A, Petkun W, et al. Estimates of current and future incidence and prevalence of atrial fibrillation in the U.S. adult population. Am J Cardiol. 2013;112(8):1142-47.

2. Go AS, Hylek EM, Phillips KA, et al. Prevalence of diagnosed atrial fibrillation in adults: national implications for rhythm management and stroke prevention: the AnTicoagulation and Risk Factors in Atrial Fibrillation (ATRIA) Study. JAMA. 2001;285(18):2370.

3. Coyne KS, Paramore C, Grandy S, et al. Assessing the direct costs of treating nonvalvular atrial fibrillation in the United States. Value Health. 2006;9(5):348-56

4. Spencer RJ, Amerena J V. Rivaroxaban in the prevention of stroke and systemic embolism in patients with non-valvular atrial fibrillation: clinical implications of the ROCKET AF Trial and its subanalyses. Am J Cardiovasc Drugs. 2015;15(6):395-401

5. Wolf PA, Abbott RD, Kannel WB. Atrial fibrillation as an independent risk factor for stroke: the Framingham Study. Stroke. 1991;22(8):983-88.

6. Ott A, Breteler MM, de Bruyne MC, et al. Atrial fibrillation and dementia in a population-based study. The Rotterdam Study. Stroke. 1997;28(2):316-21.

7. Benjamin EJ, Wolf PA, D'Agostino RB, et al. Impact of atrial fibrillation on the risk of death: the Framingham Heart Study. Circulation. 1998;98(10):946-52.

8. American Heart Association. Why atrial fibrillation (AF or AFib) matters. September 13, 2016. Available at: http://www.heart.org/HEARTORG/ Conditions/Arrhythmia/AboutArrhythmia/Why-Atrial-Fibrillation-AF-orAFib-Matters_UCM_423776_Article.jsp\#.WXQnfFGQzcs. Accessed July 22, 2017. 
9. Miyasaka Y, Barnes ME, Gersh BJ, et al. Secular trends in incidence of atrial fibrillation in Olmsted County, Minnesota, 1980 to 2000, and implications on the projections for future prevalence. Circulation. 2006;114(2):119-25.

10. Go AS, Hylek EM, Phillips KA, et al. Prevalence of diagnosed atrial fibrillation in adults. JAMA. 2001;285(18):2370-75.

11. January CT, Wann LS, Alpert JS, et al. 2014 AHA/ACC/HRS Guideline for the management of patients with atrial fibrillation: executive summary: a report of the American College of Cardiology/American Heart Association Task Force on Practice Guidelines and the Heart Rhythm Society. Circulation. 2014;130(23):2071-104.

12. Husted S, De Caterina R, Andreotti F, et al. Non-vitamin K antagonist oral anticoagulants (NOACs): no longer new or novel. Thromb Haemost. 2014;111(5):781-82.

13. Mekaj YH, Mekaj AY, Duci SB, et al. New oral anticoagulants: their advantages and disadvantages compared with vitamin $\mathrm{K}$ antagonists in the prevention and treatment of patients with thromboembolic events. Ther Clin Risk Manag. 2015;11:967-77.

14. U.S. Food and Drug Administration. FDA approves Xarelto to prevent stroke in people with common type of abnormal heart rhythm. FDA News Release. November 4, 2011. Available at: https://wayback.archive-it. org/7993/20170114035559/http://www.fda.gov/NewsEvents/Newsroom/ PressAnnouncements/ucm278646.htm. Accessed July 22, 2017.

15. U.S. Food and Drug Administration. FDA approves anti-clotting drug Savaysa. FDA News Release. January 8, 2015. Available at: https://wayback. archive-it.org/7993/20170112023821/http://www.fda.gov/NewsEvents/ Newsroom/PressAnnouncements/ucm429523.htm. Accessed July 22, 2017.

16. U.S. Food and Drug Administration. FDA approves Pradaxa to prevent stroke in people with atrial fibrillation. FDA News Release. October 19, 2010. Available at: http://www.clotcare.org/fda_approves_dabigatran.aspx. Accessed July 22, 2017.

17. U.S. Food and Drug Administration. FDA approves Eliquis to reduce the risk of stroke, blood clots in patients with non-valvular atrial fibrillation. FDA News Release. December 28, 2012. Available at: http://www. fiercebiotech.com/biotech/fda-approves-eliquis-to-reduce-risk-of-strokeblood-clots-patients-non-valvular-atrial. Accessed July 22, 2017.
18. Osterberg L, Blaschke T. Adherence to medication. N Engl J Med. 2005;353(5):487-97.

19. Kronish IM, Ye S. Adherence to cardiovascular medications: lessons learned and future directions. Prog Cardiovasc Dis. 2013;55(6):590-600.

20. Rodriguez RA, Carrier M, Wells PS. Non-adherence to new oral anticoagulants: a reason for concern during long-term anticoagulation? J Thromb Haemost. 2013;11(2):390-94.

21. Pharmacy Quality Alliance. Technical specifications for PQA approved measures. July 2014. Available at: http://www.nbch.org/nbch/files/ccLibraryFiles/Filename/000000003537/1_July2014_PQA\%20Measure\%20Manual. pdf. Accessed July 22, 2017.

22. McHorney CA, Crivera C, Laliberté F, et al. Adherence to non-vitaminK-antagonist oral anticoagulant medications based on the Pharmacy Quality Alliance measure. Curr Med Res Opin. 2015;31(12):2167-73.

23. Al-Khalili F, Lindstrom C, Benson L. Adherence to anticoagulant treatment with apixaban and rivaroxaban in a real-world setting. Clin Trials Regul Sci Cardiol. 2016;18:1-4. Available at: http://ac.els-cdn.com/ S2405587516300117/1-s2.0-S2405587516300117-main.pdf?_tid=9783. Accessed July 22, 2017

24. Yao X, Abraham NS, Sangaralingham LR, et al. Effectiveness and safety of dabigatran, rivaroxaban, and apixaban versus warfarin in nonvalvular atrial fibrillation. J Am Heart Assoc. 2016;5(6):e003725.

25. Coleman CI, Roberts M, Sobieraj D, et al. Effect of dosing frequency on chronic cardiovascular disease medication adherence. Curr Med Res Opin. 2012;28(5):669-80.

26. Srivastava K, Arora A, Kataria A, et al. Impact of reducing dosing frequency on adherence to oral therapies: a literature review and meta-analysis. Patient Prefer Adherence. 2013;7:419-34.

27. Weeda ER, Coleman CI, McHorney CA, et al. Impact of once- or twicedaily dosing frequency on adherence to chronic cardiovascular disease medications: a meta-regression analysis. Int J Cardiol. 2016;216:104-09.

28. Goldman DP, Joyce GF, Zheng Y. Prescription drug cost sharing. JAMA. 2007;298(1):61-69. 


\section{APPENDIX Multivariate Logistic Regression Modela: PDC $\geq 0.80$ at 6 Months}

Variable

\section{Cohort}

Rivaroxaban cohort

Apixaban cohort

Dabigatran cohort

Warfarin cohort

Demographics $^{c}$

Age $\geq 65$ years

Female

Regionc

South

Midwest

Northest

West

Insurance planc

$\mathrm{PPO}$

CDHP

$\mathrm{HMO}$

HAS

Indemnity/traditional

POS

Unknown

Comorbidity index scores $^{\mathrm{d}}$

Charlson Comorbidity Index score

\begin{tabular}{l}
0 \\
\hline 1 \\
$\geq 2$
\end{tabular}

$\mathrm{CHA}_{2} \mathrm{DS}_{2}$-VASc score

$\frac{1}{1}$

$\geq 2$

HAS-BLED score

$\frac{1}{1}$

$\geq 2$

Comorbidity

VTE

THA/TKA

Baseline medication $^{\mathrm{d}}$

Any oral anticoagulant at baseline

Number of different drug classes at baseline

Total baseline health care cost, \$US $2015^{\mathrm{d}}$

Index medication copayment, \$US 2015

Comorbidities, substance abuse, stress, and social risk factors associated with nonadherence ${ }^{\mathrm{d}}$

0

1

$\geq 2$

Risk factors for stroked,f

Hypertension

Diabetes

Previous stroke/TIA

Hyperlipidemia

Coronary heart disease

Heart failure

COPD
Odds Ratio (95\% CI)

Reference

$0.72(0.67-0.78)^{\mathrm{b}}$

$0.60(0.55-0.65)^{\mathrm{b}}$

$0.50(0.47-0.53)^{\mathrm{b}}$

$1.13(1.05-1.22)^{\mathrm{b}}$

$0.93(0.88-0.98)^{\mathrm{b}}$

Reference

$1.22(1.15-1.30)^{\mathrm{b}}$

$1.11(1.04-1.19)^{\mathrm{b}}$

$1.10(1.00-1.21)^{\mathrm{b}}$

Reference

$1.16(0.85-1.60)$

$0.97(0.90-1.04)$

$1.29(0.13-12.75)$

$1.08(0.97-1.20)$

$0.99(0.88-1.13)$

$1.28(1.05-1.57)^{\mathrm{b}}$

Reference $1.00(0.93-1.07)$

$1.09(1.00-1.18)^{\mathrm{b}}$

Reference

$1.17(1.04-1.31)^{\mathrm{b}}$

$1.33(1.15-1.54)^{\mathrm{b}}$

Reference 0.99 (0.88-1.11) 0.99 (0.85-1.16)

$1.05(0.95-1.15)$

$0.76(0.64-0.89)^{b}$

$1.37(1.28-1.46)^{\mathrm{b}}$

$1.00(1.00-1.01)$

$1.00(1.00-1.00)$

1.01 (1.00-1.01)

Reference

$0.99(0.93-1.05)$

$0.88(0.72-1.07)$

$0.98(0.90-1.07)$

$0.93(0.87-0.99)^{\mathrm{b}}$

$1.12(1.03-1.21)^{\mathrm{b}}$

$1.04(0.99-1.10)$

$0.91(0.86-0.97)^{\mathrm{b}}$

$0.89(0.83-0.94)^{\mathrm{b}}$

$0.98(0.90-1.06)$ 


\section{APPENDIX Multivariate Logistic Regression Modela: PDC $\geq 0.80$ at 6 Months (continued)}

Variable

Odds Ratio $(95 \% \mathrm{CI})$

Risk factors for stroked,f

Obesity

Depression

Smoking

$1.03(0.96-1.10)$

Risk factors for bleedingd,f

\begin{tabular}{l|r}
\hline Anemia & $0.92(0.86-0.99)^{\mathrm{b}}$
\end{tabular}

\begin{tabular}{l|r}
\hline Renal disease & $1.07(0.94-1.22)$ \\
\hline
\end{tabular}

Excessive fall risk (Parkinson's disease, dementia, and psychiatric disease)

CKD

Nondependent abuse of drugs

$0.88(0.80-0.97)^{\mathrm{b}}$

$0.98(0.79-1.23)$

${ }^{a}$ Model includes a fixed effect for month of index date.

${ }^{b}$ Denotes statistical significance $(P$ value $<0.05)$.

'Evaluated at index date.

dEvaluated during 6-month baseline period.

eIncludes mental disorders, substance abuse, rheumatoid arthritis, isolation, and stress.

fOnly risk factors with a proportion of patients over 5\% were adjusted for.

$C D H P=$ consumer-directed health care; $C I=$ confidence interval; $C K D=$ chronic kidney disease; $C O P D=$ chronic obstructive pulmonary disease; $H M O=$ health maintenance organization; HSA = health savings account; $P O S=$ point of service; $P P O=$ preferred provider organization; THA = total hip replacement; TIA = transient ischemic attack;

TKA = total knee replacement; VTE = venous thromboembolism. 